\title{
Restoring the valence-shell stabilization in ${ }^{140} \mathrm{Nd}$
}

\author{
R. Kern $\odot,{ }^{1, *}$ R. Zidarova, ${ }^{1,2}$ N. Pietralla, ${ }^{1}$ G. Rainovski, ${ }^{2}$ R. Stegmann, ${ }^{1}$ A. Blazhev, ${ }^{3}$ A. Boukhari, ${ }^{4}$ J. Cederkäll, ${ }^{4,5}$ \\ J. G. Cubiss, ${ }^{6}$ M. Djongolov, ${ }^{2}$ C. Fransen, ${ }^{3}$ L. P. Gaffney, ${ }^{4,7}$ K. Gladnishki, ${ }^{2}$ E. Giannopoulos, ${ }^{4,8,9}$ H. Hess, ${ }^{3}$ J. Jolie, ${ }^{3}$ \\ V. Karayonchev, ${ }^{3}$ L. Kaya, ${ }^{3}$ J. M. Keatings, ${ }^{10,11}$ D. Kocheva, ${ }^{2}$ Th. Kröll, ${ }^{1}$ O. Möller, ${ }^{1}$ G. G. O’Neill, ${ }^{12,13}$ J. Pakarinen, ${ }^{8,9}$ \\ P. Reiter, ${ }^{3}$ D. Rosiak, ${ }^{3}$ M. Scheck, ${ }^{10,11}$ J. Snall, ${ }^{5}$ P.-A. Söderström, ${ }^{1,14}$ P. Spagnoletti, ${ }^{10,11}$ M. Stoyanova, ${ }^{2}$ S. Thiel, ${ }^{3}$ A. Vogt, ${ }^{3}$ \\ N. Warr, ${ }^{3}$ A. Welker, ${ }^{4}$ V. Werner, ${ }^{1}$ J. Wiederhold, ${ }^{1}$ and H. De Witte ${ }^{15}$ \\ ${ }^{1}$ Department of Physics, Technische Universität Darmstadt, Institut für Kernphysik, 64289 Darmstadt, Germany \\ ${ }^{2}$ Faculty of Physics, St. Kliment Ohridski University of Sofia, 1164 Sofia, Bulgaria \\ ${ }^{3}$ Institut für Kernphysik, Universität zu Köln, 50937 Cologne, Germany \\ ${ }^{4}$ ISOLDE, CERN, 1211 Geneva 23, Switzerland \\ ${ }^{5}$ Department of Nuclear Physics, University of Lund, 22100 Lund, Sweden \\ ${ }^{6}$ Department of Physics, University of York, York YO10 5DD, United Kingdom \\ ${ }^{7}$ Oliver Lodge Laboratory, University of Liverpool, L69 7ZE Liverpool, United Kingdom \\ ${ }^{8}$ Helsinki Institute of Physics, University of Helsinki, P.O. Box 64, FIN-00014 Helsinki, Finland \\ ${ }^{9}$ Department of Physics, University of Jyvaskyla, P.O. Box 35, 40014 Jyvaskyla, Finland \\ ${ }^{10}$ CEPS, University of the West of Scotland, Paisley PA1 2BE, United Kingdom \\ ${ }^{11}$ SUPA, Scottish Universities Physics Alliance, United Kingdom \\ ${ }^{12}$ Department of Physics, University of Western Cape, Bellville 7535, South Africa \\ ${ }^{13}$ iThemba LABS, National Research Foundation, Somerset West 7129, South Africa \\ ${ }^{14}$ Extreme Light Infrastructure-Nuclear Physics (ELI-NP)/Horia Hulubei National Institute for R\&D in Physics \\ and Nuclear Engineering, Strasse Reactorului 30, 077125 Bucharest-Magurele, Romania \\ ${ }^{15}$ Instituut voor Kern- en Stralingsfysica, K.U. Leuven, 3001 Leuven, Belgium
}

(Received 7 August 2020; accepted 2 October 2020; published 16 October 2020)

\begin{abstract}
A projectile Coulomb-excitation experiment was performed at the radioactive-ion beam facility HIE-ISOLDE at CERN to obtain $E 2$ and $M 1$ transition matrix elements of ${ }^{140} \mathrm{Nd}$ using the multistep Coulomb-excitation code GOSIA. The absolute $M 1$ strengths, $\mathrm{B}\left(M 1 ; 2_{2}^{+} \rightarrow 2_{1}^{+}\right)=0.033(8) \mu_{\mathrm{N}}^{2}, \mathrm{~B}\left(M 1 ; 2_{3}^{+} \rightarrow 2_{1}^{+}\right)=0.26_{-0.10}^{+0.11} \mu_{\mathrm{N}}^{2}$, and $\mathrm{B}\left(M 1 ; 2_{4}^{+} \rightarrow 2_{1}^{+}\right)<0.04 \mu_{\mathrm{N}}^{2}$, identify the $2_{3}^{+}$state as the main fragment of the one-quadrupole-phonon proton-neutron mixed-symmetry state of ${ }^{140} \mathrm{Nd}$. The degree of $F$-spin mixing in ${ }^{140} \mathrm{Nd}$ was quantified with the determination of the mixing matrix element $V_{\mathrm{F}-\text { mix }}<7_{-7}^{+13} \mathrm{keV}$.
\end{abstract}

DOI: $10.1103 /$ PhysRevC.102.041304

The present day understanding of the structure of excited nuclear states is shaped by two extreme concepts, namely, those of single-particle and collective motions. The singleparticle motion reflects the quantum nature of atomic nuclei. The basic framework for understanding the single-particle motion is provided by the nuclear shell model with the spinorbital interaction [1], which leads to the existence of discrete quantum states characterized by their total $(j)$ and orbital $(l)$ angular momenta. The states are grouped in shells leading to the existence of magic numbers. The collective motion is a coherent movement of groups of few or many nucleons caused by the residual interaction, dominated by the proton-neutron interaction [2]. It reflects the many-body character of atomic

\footnotetext{
*rkern@ikp.tu-darmstadt.de
}

Published by the American Physical Society under the terms of the Creative Commons Attribution 4.0 International license. Further distribution of this work must maintain attribution to the author(s) and the published article's title, journal citation, and DOI. nuclei [3] and can geometrically be described by considering the nucleus as a homogeneous system, which vibrates or rotates [4].

In general, large-scale shell-model calculations with realistic interactions could provide a description of the collective excitations in the fundamental framework of the nuclear shell model. However, for heavy open-shell nuclei this approach runs into computational problems due to the fact that it requires enormously large configuration spaces. The interacting boson model (IBM) was introduced by Arima et al. [5] as an effective approximation used to avoid this problem. It represents a severe truncation of the shell model. Its original (sd-IBM-1) version is designed to describe quadrupolecollective excitations of even-even nuclei. The basic principle of the IBM-1 is, that the nucleons are separated in an inert core and valence nucleons. The inert core is taken to be the closest doubly magic nucleus. The valence nucleons couple pairwise to bosons and are solely responsible for the excitations of the nucleus. In the IBM-1, no distinction between valence-proton and valence-neutron bosons is made and as a result they are interchangeable. 
Besides the quantum nature and the many-body aspect, the uniqueness of the atomic nucleus as a dynamical system is also determined by its two-fluid character. This feature is taken into account in the IBM-2 [6] where separated proton and neutron bosons are introduced. The types of bosons are not always interchangeable. As a result, two distinctive classes of excitations appear. The states of the first one where proton and neutron bosons are coupled symmetrically are called full-symmetry states (FSSs) and, in fact, they are states which appear in the framework of IBM-1. The second class of states has wave functions in which, at least, one pair of proton and neutron bosons are coupled antisymmetrically. These states are called mixed-symmetry states (MSSs). The discovery of the so-called scissors mode $\left(J^{\pi}=1^{+}\right)$of the axially deformed nucleus ${ }^{156} \mathrm{Gd}$ by Bohle et al. [7] was the first experimental evidence of MSSs.

In the framework of the IBM-2, the $F$ spin is introduced to quantify the degree of coherence of the proton-boson and neutron-boson contribution of the state's wave function. The $F$ spin for bosons is analogous to isospin for nucleons. The maximum $F$ spin of a state is $F_{\max }=$ $\left(N_{\pi}+N_{v}\right) / 2$, where $N_{\pi}$ and $N_{v}$ are the number of valenceproton and valence-neutron bosons, respectively. The $F$ spin of FSSs is $F=F_{\max }$, whereas the $F$ spin of all observed MSSs is $F=F_{\max }-1$. According to the IBM-2, the mixed-symmetry one-quadrupole-phonon state $\left(2_{1, \mathrm{~ms}}^{+}\right)$is the lowest-lying MSS of heavy vibrational nuclei [8]. The isovector $(\Delta F=1)$ nature of the transition between the $2_{1, \mathrm{~ms}}^{+}$ state and the fully symmetric $2_{1}^{+}$state leads to the distinctive strong $M 1$ connection-i.e., an absolute transition strength of $\mathrm{B}\left(M 1 ; 2_{1, \mathrm{~ms}}^{+} \rightarrow 2_{1}^{+}\right) \approx 0.2 \mu_{\mathrm{N}}^{2}$ is expected [8]. In contrast, the $M 1$ strength of an isoscalar transition between FSSs $(\Delta F=$ 0 ) is highly suppressed, and the corresponding $M 1$ transition strength is an order of magnitude lower. In addition, the one-quadrupole-phonon structure of the $2_{1, \mathrm{~ms}}^{+}$state leads to a weakly collective $E 2$ transition $(\approx 1$ W.u.) (where W.u. is the Weisskopf unit) to the ground state [8].

The gradual evolution of nuclear properties as a function of the nucleon number within major shells and sudden changes, which appear at magic numbers, can well be understood in the frameworks of both collective and microscopic single-particle models. One could expect that these general trends in the collective properties within the major shells are modulated by the subshell structure. However, it is usually thought that the pairing correlations smear out and dissolve the effects from the subshell structure making them difficult to detect, especially in the case of FSSs. It has been suggested, however, that the properties of MSSs are more sensitive to the underlying subshell structure through a mechanism dubbed "valence-shell stabilization of nuclear isovector excitations" [9].

The observation of isolated $2_{1, \mathrm{~ms}}^{+}$states for the $N=80$ isotones ${ }_{52}^{132} \mathrm{Te},{ }_{54}^{134} \mathrm{Xe}$, and ${ }_{56}^{136} \mathrm{Ba}[10-12]$ was explained by the partly filled $\pi g_{7 / 2}$ orbital. This results in a valence-shell stabilization of one-quadrupole-phonon isovector excitations, but this effect disappears once the $\pi g_{7 / 2}$ orbital is fully occupied. The lack of valence-shell stabilization is manifested by a fragmentation of the one-quadrupole-phonon MSS. Indeed, a fragmented $2_{1, \mathrm{~ms}}^{+}$state is observed in ${ }^{138} \mathrm{Ce}(Z=58)$ [9]. The fragmentation implies the breaking of the $F$-spin symmetry and, consequently, a significant mixing of MSSs with FSSs. The process can be quantified by the magnitude of the mixing matrix element $\left(V_{\mathrm{F}-\mathrm{mix}}\right)$, which can be derived from the experimental data on the absolute $M 1$ and $E 2$ transition strengths of the decays of the involved states [9]. Although small matrix elements $V_{\mathrm{F}-\text { mix }}$ are derived in the cases of single isolated $2_{1, \mathrm{~ms}}^{+}$states, a large one is extracted in the case of ${ }^{138} \mathrm{Ce}$ [9].

Providing that the mechanism of valence-shell stabilization is qualitatively correct, one can expect that it will be restored for the $N=80$ isotones once the quadrupole excitations begin to develop predominantly in the $\pi d_{5 / 2}$ orbital. As a consequence of this, a single isolated one-quadrupolephonon MSS can be expected in ${ }^{140} \mathrm{Nd}$. However, it has to be noted that an unambiguous identification of MSSs in unstable nuclei can at present, solely be achieved in Coulomb excitation (CE) experiments of postaccelerated radioactive-ion beams (RIB) $[11,13,14]$. Therefore, in order to investigate this prediction of the valence-shell stabilization mechanism a projectile CE experiment was performed with the RIB of ${ }^{140} \mathrm{Nd}$ at HIE-ISOLDE at CERN $[15,16]$. In the reported CE experiment at HIE-ISOLDE at CERN, radioactive atoms were produced by bombarding a thick tantalum target with high-energy protons $(1.4 \mathrm{GeV})$ from the PS-Booster. A wide variety of isotopes was produced by the occurring fission, spallation, and fusion reactions, which were subsequently ionized by a hot surface ion source. In addition, the selective laser ionization system RILIS [17] was applied to increase the ionization rate of neodymium atoms. The first ionization process was followed by the mass selection in the General Purpose Separator and charge breeding in an electron-beam ion source REXEBIS [18]. The efficiency of the delivery of ${ }^{140} \mathrm{Nd}$ ions from the ion source to the postacceleration section was $8 \%$. The radioactive ions were postaccelerated up to 4.62 MeV/A through the REX and HIE cavities $[15,16]$ and were delivered to the Miniball [19] experimental station with a transmission of about $79 \%$. Finally, approximately $1.3 \times$ $10^{6}{ }^{140} \mathrm{Nd}$ ions per seconds impinged on a $1.5 \mathrm{mg} \mathrm{cm}^{-2}$-thick ${ }^{208} \mathrm{~Pb}$ target. The beam energy was chosen to be sufficiently low to consider the reaction of ${ }^{140} \mathrm{Nd}$ and ${ }^{208} \mathrm{~Pb}$ as "safe" $\mathrm{CE}$-i.e., the distance between the surfaces of both reaction partners was greater than $5 \mathrm{fm} \mathrm{[20].} \mathrm{The} \mathrm{scattered} \mathrm{charged}$ particles were detected by a double-sided silicon strip detector (DSSD) [19,21], which was mounted in the forward direction covering the angles between $24.0^{\circ}$ and $63.7^{\circ}$ with respect to the beam axis.

The Miniball-DSSD events were built by using the sorting code from Ref. [22]. In total, $1.2 \times 10^{6}$ single-hit and $7.8 \times$ $10^{5}$ double-hit events were recorded on the DSSD. The twodimensional histogram of the particle energy in dependence of the scattering angle (cf. Fig. 1) is used for the differentiation of beam- or targetlike particles hitting the DSSD. The twoparticle events, which involve a beam particle as well as the corresponding reaction partner (targetlike particle), are solely assigned to beamlike particle events to avoid double counting. However, single-hit targetlike events are also detected in the range where two-particle events are expected. These cases happen, if the beamlike reaction partner is lost to the interstrip boundaries of the detector, subject to pileup or otherwise not 


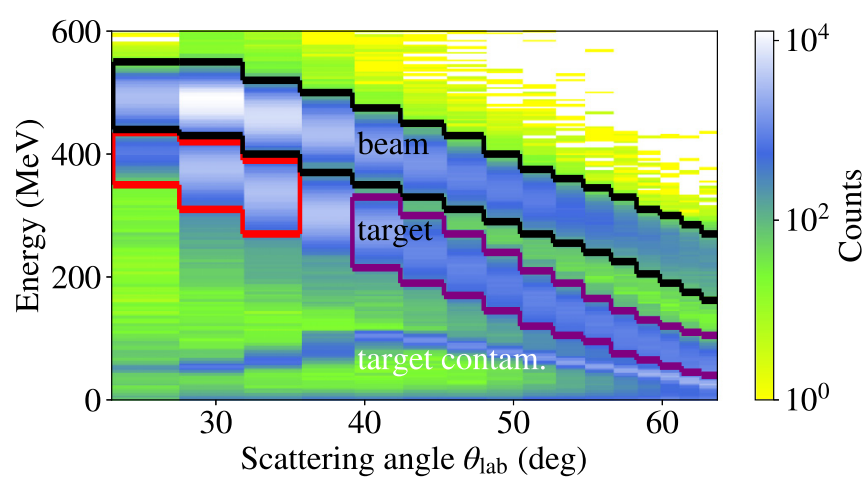

FIG. 1. The spectrum of the double-sided silicon strip detector shows the energy of the scattered heavy particles as a function of the scattering angle with respect to the beam axis (laboratory frame). Three different types of particles, heavy target, light target (contamination), and beam particles, are clearly separated. The gate for targetike particles is divided in single-hit (red) and double-hit (purple) events.

detected. Therefore, the acceptance of the single-hit target gate was limited to the three inner rings of the DSSD. Here, the beamlike reaction partner was scattered outside the angles of the DSSD. Hence, the potential corruption of the cross sections of target-gated spectra is prevented. Thus, this limitation enabled us to measure the unpeturbed CE cross sections of the target-gated and beam-gated events.

The depopulating $\gamma$ rays of the Coulomb-excited states were recorded by Miniball, in total, $1.1 \times 10^{5}$ events with the condition for a detection of a targetlike and $8.4 \times 10^{5}$ events for a beamlike recoil. Miniball consisted during the experiment of 24 sixfold segmented high-purity germanium detectors. The most prominent contamination in the $\gamma$-ray singles spectrum originated from the $\mathrm{CE}$ of ${ }^{140} \mathrm{Sm}$. The ratio of ${ }^{140} \mathrm{Sm}$ in the beam could be successfully suppressed by using RILIS to approximately $50 \%$ relative to the number of ${ }^{140} \mathrm{Nd}$ ions. The $\gamma$-ray singles spectrum is dominated by the $2_{1}^{+} \rightarrow 0_{1}^{+}$transition at $774 \mathrm{keV}$ with an energy resolution of $1.4 \%$ (full width at half maximum $=11 \mathrm{keV}$ ) after an event-by-event Doppler-shift correction, cf. Fig. 2. In total, depopulating $\gamma$ rays of three excited $2^{+}$, a $3^{-}$, three $4^{+}$and two higher-lying states with unknown spin assignment were observed, cf. Fig. 3.

The CE analysis has been performed with the multistep CE code GOSIA [23]. GOSIA reproduces the observed population yields of excited states with a given set of transition matrix elements, that are responsible for the CE, in this case, mostly $E 2$ and $E 3$ transitions. The electron conversion [24], the energy loss of the charged particles in the target [25], and the detector geometries are taken into account. The measured experimental yields are normalized to the CE cross section of the $2_{1}^{+}$state, which is determined by the known absolute E2 transition strength $\mathrm{B}\left(E 2 ; 2_{1}^{+} \rightarrow 0_{1}^{+}\right)=33.6(27) \mathrm{W} . u$. and the diagonal matrix element $\left.\left\langle 2_{1}^{+}|| E 2|| 2_{1}^{+}\right\rangle=-0.6448\right) e b$ of ${ }^{140} \mathrm{Nd}$ [26]. Knowledge about the multipole-mixing ratios of the $2_{\mathrm{i}}^{+} \rightarrow$ $2_{1}^{+}$transitions, $\delta\left(2_{2,3,4}^{+} \rightarrow 2_{1}^{+}\right)=-1.22(14),-0.08(8)$, and -0.19 (9) [27], is indispensable for the determination of the sought-for absolute $M 1$ transition strengths. The observed level scheme (cf. Fig. 3) and additional so-called buffer states, above the levels of interest, are included in the GOSIA input. The transition matrix elements are determined by a fit to the yields of both beam-gated and target-gated events, simultaneously. Both sets of experimental yields are reproduced inside their one- $\sigma$ uncertainties. These experimental data are not sensitive to the signs of the transition matrix elements except for three matrix elements involved in the excitation of the $4_{3}^{+}$state at $2400 \mathrm{keV},\left\langle 2_{1}^{+}\|E 2\| 2_{2}^{+}\right\rangle,\left\langle 2_{1}^{+}|| E 2|| 4_{3}^{+}\right\rangle$, and $\left\langle 2_{2}^{+}\|E 2\| 4_{3}^{+}\right\rangle$, and the ground-state matrix element of the $3_{1}^{-}$ state $\left\langle 0_{1}^{+}\|E 3\| 3_{1}^{-}\right\rangle$. This leads to several reasonable solutions, which results in additional systematical uncertainties of all matrix elements. These are included in the total uncertainties together with the statistical uncertainties. The resulting $M 1, E 2$, and $E 3$ transition strengths are shown in Table I.

The most promising candidate for the main fragment of the $2_{1, \mathrm{~ms}}^{+}$state is the $2_{3}^{+}$state. Some 113 and $12 \gamma$-ray events of its predominant decay to the $2_{1}^{+}$state at $1366 \mathrm{keV}$ are observed in the beam-particle-gated $\gamma$-singles and coincidence spectrum, respectively. Furthermore, $33 \gamma$-ray events of the

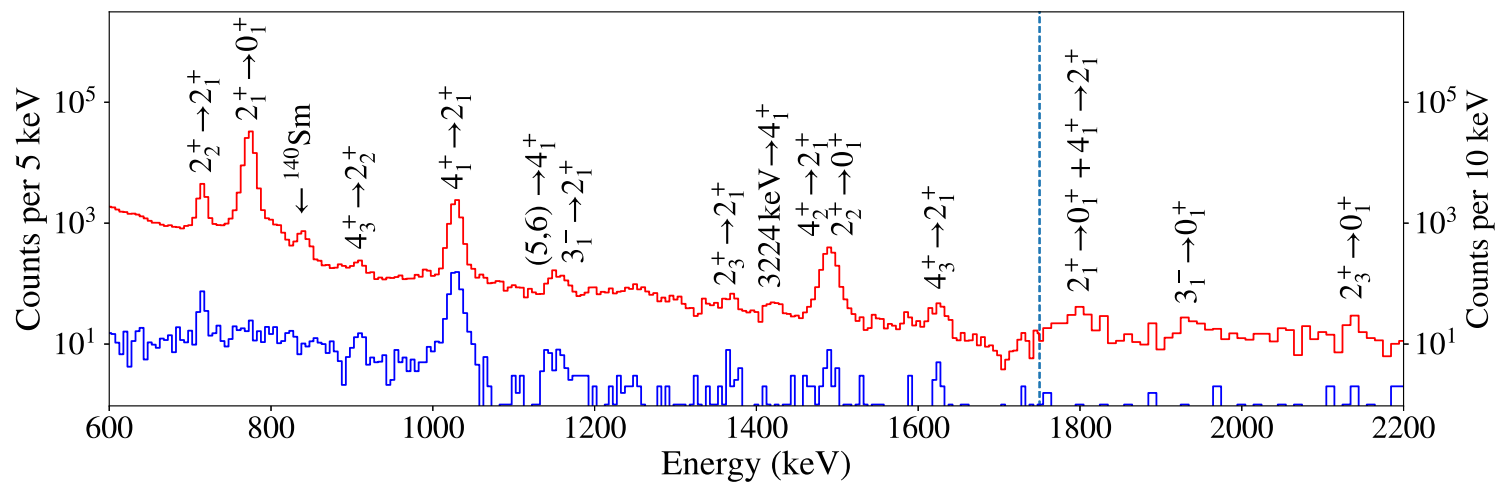

FIG. 2. The time-random background subtracted, Doppler-corrected, beam-particles gated $\gamma$-ray singles spectrum (red) and the $\gamma$-ray coincidence spectrum (blue) with an additional gate on the $2_{1}^{+} \rightarrow 0_{1}^{+}$transition of ${ }^{140} \mathrm{Nd}$. Peaks are marked with the identified transition emitted following the CE of ${ }^{140} \mathrm{Nd}$. Additionally, a peak at $840 \mathrm{keV}$ was identified as a transition of the beam contaminant ${ }^{140} \mathrm{Sm}$, which vanishes in the $\gamma$-ray coincidence spectrum. The binning changes after the dashed line for visibility. 
TABLE I. Measured properties of the levels and $\gamma$-ray transitions of ${ }^{140} \mathrm{Nd}$. Level energies and spin assignments are adopted from Ref. [28] unless otherwise noted. The relative $\gamma$-ray intensities determined from the beam-gated (expt. 1) and the target-gated (expt. 2) $\gamma$-ray spectra are corrected for efficiency.

\begin{tabular}{|c|c|c|c|c|c|c|c|c|}
\hline $\begin{array}{l}E_{\text {level }} \\
(\mathrm{keV})\end{array}$ & $J^{\pi}$ & $\begin{array}{c}E_{\gamma} \\
(\mathrm{keV})\end{array}$ & $J_{f}^{\pi}$ & $\begin{array}{c}I_{\gamma} \\
(\operatorname{expt.1)})\end{array}$ & $\begin{array}{c}I_{\gamma} \\
\text { (expt. 2) }\end{array}$ & $\begin{array}{c}\delta \\
\text { from Ref. [27] }\end{array}$ & $\pi \lambda$ & $\mathrm{B}(\pi \lambda) \downarrow^{\mathrm{a}}$ \\
\hline 774 & $2_{1}^{+}$ & 774 & $0_{1}^{+}$ & $100(1)$ & $100(1)$ & & $E 2$ & $33.6(27)^{\mathrm{b}}$ \\
\hline \multirow[t]{3}{*}{1490} & $2_{2}^{+}$ & 1490 & $0_{1}^{+}$ & $2.1(1)$ & $5.5(6)$ & & $E 2$ & $1.76(13)$ \\
\hline & & $716^{\mathrm{d}}$ & $2_{1}^{+}$ & $1.6(2)$ & $2.7(8)$ & $-1.22(14)$ & $E 2$ & $33(3)$ \\
\hline & & & & & & & $M 1$ & $0.033(8)$ \\
\hline \multirow[t]{2}{*}{1936} & $3_{1}^{-}$ & 1936 & $0_{1}^{+}$ & $0.08(2)$ & & & $E 3$ & $6(2)$ \\
\hline & & $1162^{\mathrm{d}}$ & $2_{1}^{+}$ & $0.25(4)$ & $0.9(4)$ & & & \\
\hline \multirow[t]{3}{*}{2140} & $2_{3}^{+}$ & 2140 & $0_{1}^{+}$ & $0.07(2)$ & & & $E 2$ & $1.9(6)$ \\
\hline & & $1366^{\mathrm{d}}$ & $2_{1}^{+}$ & $0.19(3)$ & & $-0.08(8)$ & $E 2$ & $0.3_{-0.3}^{+0.6}$ \\
\hline & & & & & & & $M 1$ & $0.26_{-0.10}^{+0.11}$ \\
\hline \multirow[t]{2}{*}{2400} & $4_{3}^{+}$ & $1626^{\mathrm{d}}$ & $2_{1}^{+}$ & $0.21(4)$ & & & $E 2$ & $1.6(3)$ \\
\hline & & $910^{\mathrm{d}}$ & $2_{2}^{+}$ & $0.31(5)$ & & & $E 2$ & $41(7)$ \\
\hline $2950^{f}$ & $(5,6)^{\mathrm{e}}$ & $1148^{\mathrm{gh}}$ & $4_{1}^{+}$ & $0.40(6)$ & $1.6(5)$ & & $E 2$ & $30(3)$ \\
\hline $3224^{\mathrm{f}}$ & & $1422^{g h}$ & $4_{1}^{+}$ & $0.17(3)$ & $1.0(2)$ & & $E 2$ & $22(4)$ \\
\hline
\end{tabular}

${ }^{\mathrm{a}} B(M 1)$ values are given in $\mu_{N}^{2}, B(E 2)$, and $B(E 3)$ values are given in W.u. $\left(E 2: 1\right.$ W.u. $=4.32 \times 10^{-3} e^{2} \mathrm{~b}^{2} E 3: 1 \mathrm{~W}$. u. $\left.=1.16 \times 10^{3} e^{2} \mathrm{~b}^{3}\right)$.

${ }^{\mathrm{b}}$ Transition strength adopted from Ref. [26].

${ }^{\mathrm{c}}$ This value is extracted without considering $E 0$ excitation. It has to be considered as an estimate only.

${ }^{\mathrm{d}}$ Observed in coincidence spectrum of the $2_{1}^{+} \rightarrow 0_{1}^{+}$transition.

${ }^{\text {e }}$ Spin adopted from Ref. [29].

${ }^{\mathrm{f}}$ Assumed as a $6^{+}$state in the GOSIA analysis

${ }^{\mathrm{g}}$ Observed in coincidence spectra of the $4_{1}^{+} \rightarrow 2_{1}^{+}$and $2_{1}^{+} \rightarrow 0_{1}^{+}$transitions.

${ }^{\mathrm{h}}$ Transition energy adopted from Ref. [30].

ground-state transition at $2140 \mathrm{keV}$, which was unobserved before, were counted (cf. Fig. 2). A $\chi^{2}$-surface analysis has been performed to obtain the correlation of the two $E 2$ transition matrix elements, which are primarily responsible for the $\mathrm{CE}$ of the $2_{3}^{+}$state, $\left\langle 0_{1}^{+}\|E 2\| 2_{3}^{+}\right\rangle$and its diagonal matrix element $\left\langle 2_{3}^{+}\|E 2\| 2_{3}^{+}\right\rangle$, cf. Fig. 4. The same diagonal $E 2$ matrix element of the $2_{3}^{+}$state as it is known for the $2_{1}^{+}$state is applied as an additional data point to the analysis. The $\chi^{2}$-surface distribution in Fig. 4 shows the strong correlation of these two

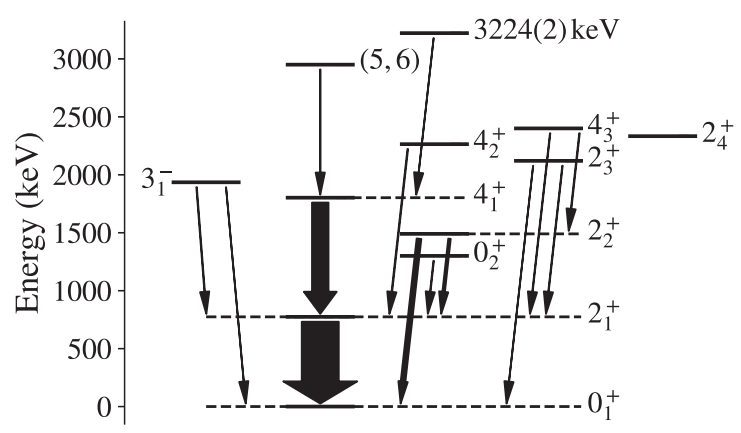

FIG. 3. The partial level scheme of ${ }^{140} \mathrm{Nd}$. The widths of the transitions correspond to the observed intensities. The $2_{1}^{+} \rightarrow 0_{1}^{+}$ transition width is scaled down by a factor of 5 for visibility. matrix elements and it is used to determine the uncertainty of $\left\langle 0_{1}^{+}\|E 2\| 2_{3}^{+}\right\rangle$.

The resulting matrix element $\left\langle 0_{1}^{+}\|E 2\| 2_{3}^{+}\right\rangle=$ $0.204(34) e b$, the branching ratio of the depopulating $\gamma$-ray transitions of the $2_{3}^{+}$state $\mathrm{I}_{2_{3}^{+} \rightarrow 0_{1}^{+}} / \mathrm{I}_{2_{3}^{+} \rightarrow 2_{1}^{+}}=0.38(11)$, and the multipole-mixing ratio [27] lead to the absolute $M 1$ transition strength of $\mathrm{B}\left(M 1 ; 2_{3}^{+} \rightarrow 2_{1}^{+}\right)=0.26(11) \mu_{\mathrm{N}}^{2}$. The

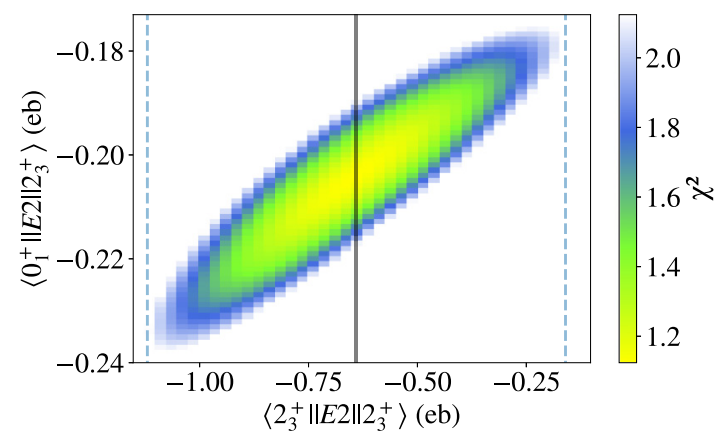

FIG. 4. $\chi^{2}$-surface distribution with respect to the diagonal and transitional matrix elements of the $2_{3}^{+}$state. The dashed gray lines indicate the limits of the diagonal matrix element of the $2_{1}^{+}$state, which is applied to the $2_{3}^{+}$state. 


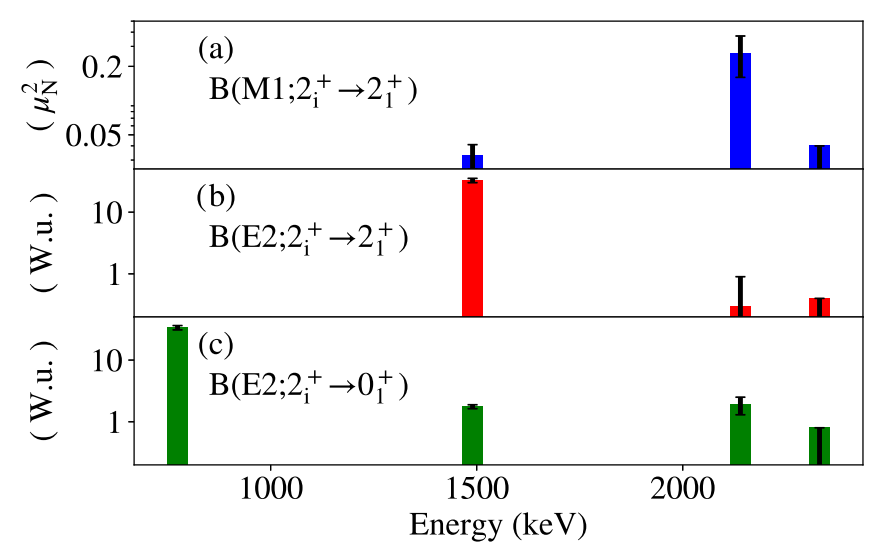

FIG. 5. The values for $\mathrm{B}\left(M 1 ; 2_{\mathrm{i}}^{+} \rightarrow 2_{1}^{+}\right), \mathrm{B}\left(E 2 ; 2_{\mathrm{i}}^{+} \rightarrow 2_{1}^{+}\right)$, and $\mathrm{B}\left(E 2 ; 2_{\mathrm{i}}^{+} \rightarrow 0_{1}^{+}\right)$strengths of ${ }^{140} \mathrm{Nd}$ are displayed in (a)-(c), respectively.

lower uncertainty is reduced by the known upper limit $\mathrm{I}_{2_{3}^{+} \rightarrow 0_{1}^{+}} / \mathrm{I}_{2_{3}^{+} \rightarrow 2_{1}^{+}}<0.42$ [27]. The analogous procedure has been conducted for the $2_{4}^{+}$state. Decays of the $2_{4}^{+}$state are unobserved in this experiment, hence, an upper limit of $\mathrm{B}\left(M 1 ; 2_{4}^{+} \rightarrow 2_{1}^{+}\right)<0.04 \mu_{\mathrm{N}}^{2}$ is deducted by assuming an intensity of the $2_{4}^{+} \rightarrow 2_{1}^{+}$and $2_{4}^{+} \rightarrow 0_{1}^{+}$transitions at the edge of the detection limit. The known branching ratio $\mathrm{I}_{2_{4}^{+} \rightarrow 0_{1}^{+}} / \mathrm{I}_{2_{4}^{+} \rightarrow 2_{1}^{+}}=1.9(6)$ [31] is applied to the calculation. The resulting $M 1$ - and $E 2$-strength distributions are displayed in Fig. 5.

Various IBM-2, large-scale shell model (LSSM), and quasiparticle phonon model (QPM) calculations were carried out to reproduce and predict the $M 1$-strength evolution of the $N=80$ isotones [32-34]. Using a modified pairing strength, Sieja et al. [33] succeeded in accurately describing the $M 1$ strengths of the MSSs of $N=80$ isotones from ${ }^{132} \mathrm{Te}$ to ${ }^{138} \mathrm{Ce}$, whereas predicting an increase in the $M 1$ strength from ${ }^{138} \mathrm{Ce}$ to ${ }^{140} \mathrm{Nd}$. The model predicted a $\mathrm{B}\left(M 1 ; 2_{\mathrm{MSS}}^{+} \rightarrow 2_{1}^{+}\right) \approx 0.2 \mu_{\mathrm{N}}^{2}$ at around $2.3 \mathrm{MeV}$ (cf. Fig. 6) for ${ }^{140} \mathrm{Nd}$.

The $2_{3,4}^{+}$states of ${ }^{140} \mathrm{Nd}$ at 2.140 and $2.332 \mathrm{MeV}$, respectively, were experimentally identified as the two fragments of the $2_{1, \mathrm{~ms}}^{+}$state due to their predominant $M 1$ transitions to the $2_{1}^{+}$state [27]. The result of $\mathrm{B}\left(M 1 ; 2_{3}^{+} \rightarrow 2_{1}^{+}\right)=0.26_{-0.10}^{+0.11} \mu_{\mathrm{N}}^{2}$ indicates that the $2_{3}^{+}$state is the main fragment of the MSS. This is also in good agreement with the theoretical predictions from Ref. [33] (LSSM) and from Ref. [30] (QPM). Hence, the $2_{4}^{+}$state can be adopted, at most, as the minor fragment of the $2_{1, \mathrm{~ms}}^{+}$state.

The $F$-spin mixing matrix element is determined to quantify the degree of mixing of the $F$ spin in ${ }^{140} \mathrm{Nd}$. The simplest scenario is a two-state mixing where the $M 1$ strength between two FSSs is zero, according to the IBM-2. However, the concept of idealized FSSs is an approximation and does not reflect reality. Therefore the $M 1$ connection between nuclear states, which are labeled as FSSs, is not strictly forbidden, although it is highly suppressed. This general nonzero $M 1$ strength between FSSs is determined by the $\mathrm{B}\left(M 1 ; 2_{2}^{+} \rightarrow\right.$ $\left.2_{1}^{+}\right)=0.033(8) \mu_{\mathrm{N}}^{2}$ assuming the $2_{2}^{+}$state as a pure FSS. Thus, a more realistic mixing scenario is applied where the $M 1$ strength between FSSs interferes constructively with the $M 1$

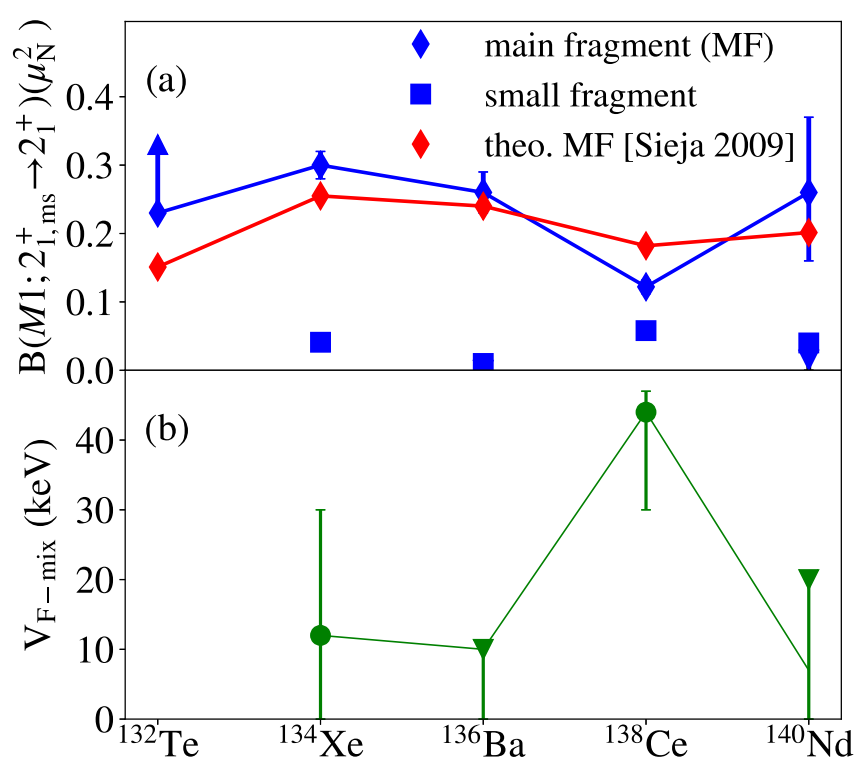

FIG. 6. The $M 1$ transition strengths $\mathrm{B}\left(M 1 ; 2_{\mathrm{i}}^{+} \rightarrow 2_{1}^{+}\right)$of the main and the small fragment of the $2_{1, \mathrm{~ms}}^{+}$state of the $N=80$ isotones are compared to a theoretical $\mathrm{B}\left(M 1 ; 2_{1, \mathrm{~ms}}^{+} \rightarrow 2_{1}^{+}\right)$systematic (Sieja et al., Ref. [33]) in (a). The resulting $F$-spin mixing matrix elements (o) and upper limits $(\nabla)$ are shown in (b). $V_{\mathrm{F}-\text { mix }}$ of ${ }^{136} \mathrm{Ba}$ and ${ }^{138} \mathrm{Ce}$ are taken from Ref. [9].

strength between a MSS and a FSS. This results in a $F$-spin mixing matrix element of $V_{\mathrm{F}-\text { mix }}<7_{-7}^{+13} \mathrm{keV}$. This clearly indicates a decrease in the strength of the $F$-spin mixing from ${ }^{138} \mathrm{Ce}$ to ${ }^{140} \mathrm{Nd}$, in contrast to the calculations $\left[\mathrm{V}_{\mathrm{F}-\text { mix }}=\right.$ 92(14) keV] from Ref. [27], which were solely based on multipole-mixing ratios. This decrease displays the observed evolution of these isotones' $\mathrm{B}\left(M 1 ; 2_{\mathrm{i}}^{+} \rightarrow 2_{1}^{+}\right)$distributions, cf. Fig. 6: the ratio of the $M 1$ strengths of the small fragment and the main fragment in ${ }^{138} \mathrm{Ce}$ is approximately $1 / 2$ whereas it is less than $1 / 8$ in ${ }^{140} \mathrm{Nd}$. So the $F$-spin mixing is obviously reduced again when passing the $Z=58$ subshell closure.

Looking at the evolution of $V_{\mathrm{F}-\text { mix }}$ for the $N=80$ isotones (cf. Fig. 6), the strong mixing of ${ }^{138} \mathrm{Ce} V_{\mathrm{F}-\text { mix }}=$ $44(3)_{-11}^{+4} \mathrm{keV}$ [9] clearly stands out. The $V_{\mathrm{F}-\text { mix }}$ of ${ }^{134} \mathrm{Xe}$ is calculated under the same assumptions as in ${ }^{140} \mathrm{Nd}$, namely, a $M 1$ strength between FSSs of $\mathrm{B}\left(M 1 ; 2_{2}^{+} \rightarrow 2_{1}^{+}\right)=$ $0.033(8) \mu_{\mathrm{N}}^{2}$, and with spectroscopic information taken from Ref. [10]. This results in $V_{\mathrm{F}-\text { mix }}=12_{-12}^{+18} \mathrm{keV}$ for ${ }^{134} \mathrm{Xe}$. The evolution of the fragmentation of the MSSs from ${ }^{134} \mathrm{Xe}$ to ${ }^{140} \mathrm{Nd}$ shows a restauration of the valence-shell stabilization for the even-even $N=80$ isotones at ${ }^{140} \mathrm{Nd}$ and indicates the $F$ spin as a well-conserved quantum number for the $N=80$ isotones apart from the subshell closure at $Z=58$.

To summarize, a projectile CE experiment was performed at HIE-ISOLDE at CERN to determine absolute $M 1$ transition strengths $\mathrm{B}\left(M 1 ; 2_{\mathrm{i}}^{+} \rightarrow 2_{1}^{+}\right)$of ${ }^{140} \mathrm{Nd}$ to identify the one-quadrupole-phonon $2_{1, \mathrm{~ms}}^{+}$state. In total, $14 \gamma$-ray transitions were observed, ten of them additionally in coincidence spectra, and assigned to the decay of ten excited states of ${ }^{140} \mathrm{Nd}$. The identifications of the $2_{3}^{+}$state as the main fragment and the $2_{4}^{+}$state, at most, as the 
small fragment of the $2_{1, \mathrm{~ms}}^{+}$state of ${ }^{140} \mathrm{Nd}$ is based on the determined absolute $M 1$ transition strengths, $\mathrm{B}\left(M 1 ; 2_{3}^{+} \rightarrow\right.$ $\left.2_{1}^{+}\right)=0.26_{-0.10}^{+0.11} \mu_{\mathrm{N}}^{2}, \mathrm{~B}\left(M 1 ; 2_{2}^{+} \rightarrow 2_{1}^{+}\right)=0.033(8) \mu_{\mathrm{N}}^{2}, \quad$ and $\mathrm{B}\left(M 1 ; 2_{4}^{+} \rightarrow 2_{1}^{+}\right)<0.04 \mu_{\mathrm{N}}^{2}$. The corresponding $F$-spin mixing matrix element $V_{\mathrm{F}-\text { mix }}<7_{-7}^{+13} \mathrm{keV}$ shows a significant decrease in the $F$-spin mixing strength from ${ }^{138} \mathrm{Ce}$ to ${ }^{140} \mathrm{Nd}$. This evolution and the $M 1$ strength concentrated to a large extent in the $2_{3}^{+} \rightarrow 2_{1}^{+}$transition indicate the restoration of the valence-shell stabilization after the subshell closure at $Z=58$ for the $N=80$ isotones at ${ }^{140} \mathrm{Nd}(Z=60)$.
We gratefully acknowledge the support of the staff members at CERN during the experimental runs. This work was supported by the BMBF Grants No. 05P18RDCIA, No. 05P19RDFN1, No. 05P18RDFN9, and No. 05P18PKCIA, by the BgNSF Grant No. DN08/23/2016, by Grants from the U.K. Science and Technology Facilities Council Grants No. ST/R004056/1, No. ST/P005101/1, and No. ST $/$ P003885/1, the Academy of Finland (Finland) Grant No. 307685, and the European Union within the Horizon 2020 Research and Innovation Program No. 654002 (ENSAR-2) and No. 665779 (COFUND).
[1] M. G. Mayer, Phys. Rev. 78, 16 (1950).

[2] R. F. Casten, Phys. Lett. 152B, 145 (1985).

[3] T. Otsuka, Y. Tsunoda, T. Abe, N. Shimizu, and P. Van Duppen, Phys. Rev. Lett. 123, 222502 (2019).

[4] A. Bohr and B. Mottelson, Nuclear Structure (Benjamin, Reading, MA, 1975), Vol. II.

[5] A. Arima, T. Ohtsuka, F. Iachello, and I. Talmi, Phys. Lett. 66B, 205 (1977).

[6] F. Iachello, Phys. Rev. Lett. 53, 1427 (1984).

[7] D. Bohle, A. Richter, W. Steffen, A. E. L. Dieperink, N. LoIudice, F. Palumbo, and O. Scholten, Phys. Lett. 137B, 27 (1984).

[8] N. Pietralla, P. von Brentano, and A. F. Lisetskiy, Prog. Part. Nucl. Phys 60, 225 (2008).

[9] G. Rainovski, N. Pietralla, T. Ahn, C. J. Lister, R. V. F. Janssens, M. P. Carpenter, S. Zhu, and C. J. Barton, Phys. Rev. Lett. 96, 122501 (2006).

[10] T. Ahn, L. Coquard, N. Pietralla, G. Rainovski, A. Costin, R. V. F. Janssens, C. J. Lister, M. P. Carpenter, S. Zhu, and K. Heyde, Phys. Lett. B 679, 19 (2009).

[11] M. Danchev, G. Rainovski, N. Pietralla, A. Gargano, A. Covello, C. Baktash et al., Phys. Rev. C 84, 061306(R) (2011).

[12] N. Pietralla, D. Belic, P. von Brentano, C. Fransen, R.-D. Herzberg, U. Kneissl, H. Maser, P. Matschinsky, A. Nord, T. Otsuka, H. H. Pitz, V. Werner, and I. Wiedenhöver, Phys. Rev. C 58, 796 (1998).

[13] K. Moschner, A. Blazhev, J. Jolie, N. Warr, P. Boutachkov, P. Bednarczyk et al., Phys. Rev. C 94, 054323 (2016).

[14] V. Vaquero, A. Jungclaus, P. Doornenbal, K. Wimmer, A. M. Moro, K. Ogata et al., Phys. Rev. C 99, 034306 (2019).

[15] M. J. G. Borge and K. Riisager, Eur. Phys. J. A 52, 334 (2016).

[16] Y. Kadi, Y. Blumenfeld, W. Venturini Delsolaro, M. A. Fraser, M. Huyse, A. Papageorgiou Koufidou, J. A. Rodriguez, and F. Wenander, J. Phys. G: Nucl. Part. Phys. 44, 084003 (2017).

[17] V. N. Fedosseev, L.-E. Berg, N. Lebas, O. J. Launila, M. Lindroos, R. Losito, B. A. Marsh, F. K. Österdahl, T. Pauchard, G. Tranströmer, and J. Vannesjö, Nucl. Instrum. Methods Phys. Res., Sect. B 266, 4378 (2008).
[18] F. Wenander, J. Instrument. 5, C10004 (2010).

[19] N. Warr, J. Van de Walle, M. Albers, F. Ames, B. Bastin, C. Bauer et al., Eur. Phys. J. A 49, 40 (2013).

[20] D. Cline, Annu. Rev. Nucl. Part. Sci. 36, 683 (1986).

[21] A. N. Ostrowski, S. Cherubini, T. Davinson, D. Groombridge, A. M. Laird, A. Musumarra, A. Ninane, A. di Pietro, A. C. Shotter, and P. J. Woods, Nucl. Instrum. Methods Phys. Res., Sect. A 480, 448 (2002).

[22] L. P. Gaffney and J. Konki, MiniballCoulexSort [https://doi.org/ 10.5281/zenodo.1344355] (2019).

[23] T. Czosnyka, D. Cline, and C. Y. Whu, Bull. Am. Phys. Soc. 28, 745 (1983); GOSIA user manual, http://www.pas.rochester. edu/ cline/Gosia/Gosia_Manual_20120510.pdf, accessed June 2020.

[24] T. Kibédi, T. W. Burrows, M. B. Trzhaskovskaya, P. M. Davidson, and C. W. Nestor, Jr., Nucl. Instrum. Methods Phys. Res., Sect. A 589, 202 (2008).

[25] J. F. Ziegler, M. D. Ziegler, and J. P. Biersack, Nucl. Instrum. Methods Phys. Res., Sect. B 268, 1823 (2010).

[26] C. Bauer, G. Rainovski, N. Pietralla, D. Bianco, A. Blazhev, T. Bloch et al., Phys. Rev. C 88, 021302(R) (2013).

[27] E. Williams, R. J. Casperson, V. Werner, H. Ai, P. Boutachkov, M. Chamberlain, G. Gürdal, A. Heinz, E. A. McCutchan, J. Qian, and R. Winkler, Phys. Rev. C 80, 054309 (2009).

[28] N. Nica, Nucl. Data Sheets 154, 1 (2018).

[29] R. J. Casperson, Experimental and numerical analysis of mixedsymmetry states and large boson systems, Dissertation, Yale University, 2010.

[30] K. A. Gladnishki, G. Rainovski, P. Petkov, J. Jolie, N. Pietralla, A. Blazhev et al., Phys. Rev. C 82, 037302 (2010).

[31] G. G. Kennedy, S. C. Gujrathi, and S. K. Mark, Z. Phys. A 274, 233-239 (1975).

[32] D. Bianco, F. Andreozzi, N. Loludice, A. Porrino, and F. Knapp, Phys. Rev. C 85, 034332 (2012).

[33] K. Sieja, G. Martinez-Pinedo, L. Coquard, and N. Pietralla, Phys. Rev. C 80, 054311 (2009).

[34] N. Lo Iudice, C. Stoyanov, and D. Tarpanov, Phys. Rev. C 77, 044310 (2008). 